\title{
G4-Selective Ligand Induced Autophagy
}

\author{
Piyali Majumder*a ${ }^{* a}$ Chinmayee Shukla ${ }^{a}$ and Bhaskar Datta*a.b
}

\author{
a. Department of Biological Engineering, Indian Institute of Technology, Gandhinagar, Gujarat, India \\ b. Department of Chemistry, Indian Institute of Technology, Gandhinagar, Gujarat, India. \\ * Corresponding authors \\ $\dagger$ Bhaskar Datta, Department of Chemistry \& Biological Engineering, Indian Institute of Technology Gandhinagar, Palaj, \\ Gandhinagar 382355 India. \\ Telephone: 079-2395-2427; E-mail: bdatta@iitgn.ac.in. \\ $\dagger$ Piyali Majumder, Department of Biological Engineering, Indian Institute of Technology Gandhinagar, Palaj, Gandhinagar \\ 382355 India. \\ E-mail: piyali.majumder@iitgn.ac.in
}

G-quadruplex (G4) structures have emerged as singular therapeutic targets for cancer and neurodegeneration. Autophagy is a housekeeping cellular homeostatic mechanism and deregulation of autophagy is common in cancer and in neurodegenerative diseases. In this study, we identified the presence of 46 putative G4 sequences in the MTOR gene by use of QGRS mapper tool. We sought to connect these putative G4 sequences to a functional context by leveraging G4-targeting ligands. A G4-selective dimeric carbocyanine dye Bis-4,3 and the porphyrin TMPyP4 were used to affect the replication, transcription and translation of the MTOR gene. The ligand-induced induction of autophagic pathway via MTOR gene regulation was monitored upon treatment of HeLa and SHSY-5Y cells with G4-targeting ligands. The use of Bis-4,3 was compared with the known G4-stabilizing activity of TMPyP4. Our results show that treatment with G4-selective ligands downregulates mTOR activity and leads to the induction of excessive autophagy. This is first report on effect of G4-selective ligands on MTOR regulation and mTOR expression. mTOR being the key negative regulator of autophagy, the current work suggests potential of G4 stabilizing ligands towards induction of autophagy through the downregulation of mTOR.

Keywords: G-Quadruplex, mTOR, TMPyP4, Bis-4,3, Autophagy

\section{Introduction}

The perceptions surrounding G-quadruplexes (G4) have undergone a significant makeover over the past two decades. While originally viewed as unusual structures that could be produced in vitro confinement, the past two decades have witnessed mounting evidence on their physiological relevance. Computational analysis has identified over 350,000 putative quadruplex forming sequences within the human genome ${ }^{1}$. Thermodynamic and kinetic studies of DNA Gquadruplexes reveal higher stability and slower unfolding in comparison to double stranded DNA ${ }^{2}$. In vivo studies have identified the presence of G-quadruplex structures using specific antibodies ${ }^{3}$. G-quadruplex structures have been experimentally mapped in human genomic DNA and are found to be enriched at promoter sites, $5^{\prime}$-UTRs, first introns and gene $3^{\prime}$ regions suggesting deep involvement in gene regulatory events ${ }^{4}$. As a corollary, G4 has emerged as attractive therapeutic targets for several disease conditions and disorders ${ }^{5}$.

G4 associated with genes such as c-MYC, NRAS, KRAS, BCL-2 and c-KIT have been implicated in modulating their transcription and translation ${ }^{6-8}$. Small molecule-mediated stabilization of such G4 elements elicits transcriptional control that ultimately halts tumour progression. G4-stabilization in oncogene promoter regions has been established as an effective mechanism for down-regulation of genes involved in tumour survival and metastasis ${ }^{9}$. G4-targeting appears to be a promising anti-cancer approach also because of the over-expression of putative quadruplex containing genes in specific cancers ${ }^{10}$. Small molecule-mediated stabilization of these and other G4s have been shown to affect global processes such as apoptosis, autophagy, senescence and signalling pathways such as hypoxia-inducible factor pathway ${ }^{11,12}$.

Autophagy is characterized by sequestration and digestion of cellular components in a double membrane vesicle, to maintain a homeostatic balance ${ }^{13}$. The initiation of autophagy is controlled by the ULK1 (Unc-51 like autophagy activating kinase) complex and integrates stress signals from mTOR (mechanistic target of rapamycin) complex 1 (mTORC1) ${ }^{14}$. mTOR functions as the most crucial antagonistic regulator of autophagy ${ }^{15-17}$. Autophagy generates stress response that supports the tumour cells to survive in the nutrient deficient hypoxic microenvironment ${ }^{18}$. However, it can also act as tumour suppressor by preventing the accumulation of damaged proteins and organelles while displaying its Janus-faced behaviour ${ }^{19}$. Several autophagy inducers are used so far as therapeutics for cancer provided that show chemo sensitivity in apoptosis incompetent cancer cells ${ }^{20}$.

It has recently been reported that excessive autophagy can trigger caspase independent and LMP (lysosomal membrane permeability)-cathepsin dependent type II program cell death (Autophagic Cell Death; ACD) through Bax (Bcl-2 associated X 
protein) and Bak (Bcl-2 homologous antagonist/killer) proteins ${ }^{21}$. Thus, inducing excess autophagy in a tumour cell can lead to cell death via ACD pathway. While small molecule mediated quadruplex stabilization has shown to induce autophagy, the exact mechanism by which this occurs remains unknown ${ }^{22,23}$. Notably, mTOR maintains the balance between cell growth and autophagy by responding to stress, growth factors, and nutrition levels ${ }^{24}$. We identified 46 potential quadruplex forming regions in the MTOR gene by using QGRS mapper ${ }^{25}$.

In this work, we report the presence of a novel G-quadruplex in the MTOR gene, a key negative regulator of autophagy. We demonstrate the effect of G4-stabilizing ligands Bis-4,3 and TMPyP4 towards suppression of mTOR expression leading to induction of excessive autophagy, followed by cell death through the ACD pathway.

\section{Results and Discussion:}

The abundance of G-quadruplex structures in MTOR gene

mTOR is the critical regulator of various metabolic pathways inside the cell ${ }^{17}$. MTOR gene is localized on chromosome 1 . We used the QGRS mapper tool to measure putative Gquadruplex (G4) forming sites ${ }^{25}$.

This bioinformatics analysis was performed by using the initial $30 \mathrm{~kb}$ sequence from the RefSeqGene (LRG_734) for MTOR gene (NM_004958.4) on chromosome 1. The 30kb stretch includes the upstream region, the initial eleven intronic regions and twelve exonic regions of MTOR genes. The QGRS mapper analysis indicated 150 putative sites for this $30 \mathrm{~kb}$ sequence [Supplementary Data 1]. On the other hand, the cDNA sequence of MTOR gene showed 46 putative sites [Supplementary Data 2]. The G- rich regions are situated in the upstream of MTOR gene and are displayed in the Supplementary Data 1. Upstream elements have conventionally been implicated in regulation of replication or transcription of genes. In the MTOR gene, three G-rich sites emerge from the QGRS bioinformatics analysis. These three G-rich sites are at $5 \mathrm{~Kb}, 8 \mathrm{~Kb}$ and $14 \mathrm{~kb}$ from nucleotide 1 [Fig. 1A and Supplementary Data 1]. Among these three G-rich regions, $5 \mathrm{~Kb}$ region is an upstream region, $8 \mathrm{~Kb}$ is around gene start site (ATG) and $14 \mathrm{~Kb}$ is a downstream region on TOR gene. It is well known that secondary structure in the upstream region of a gene modulates the attachment of polymerases on the DNA strand, which effectively attune the replication or transcription of the gene. The presence of putative Gquadruplex forming sequences in the upstream region or the start-site-adjacent region suggests a distinctive role of G4 in the expression of MTOR gene.

We investigated quadruplex-forming potential of the oligos corresponding to putative G4-forming sequences identified by QGRS mapper analysis via Circular Dichroism (CD) spectroscopy. CD is capable of informing about the existence

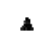

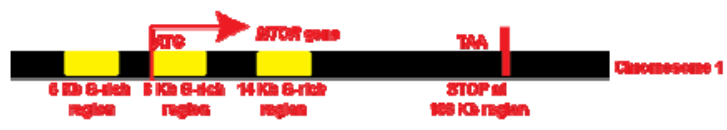

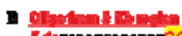

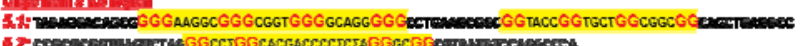

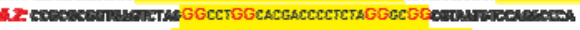

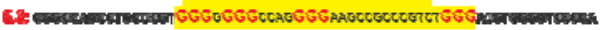

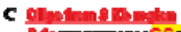

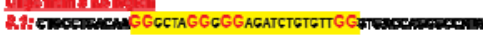

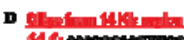

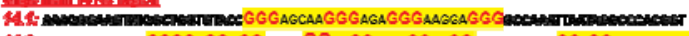

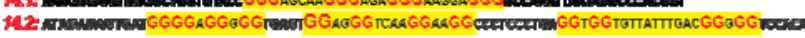

$\mathbf{z}$
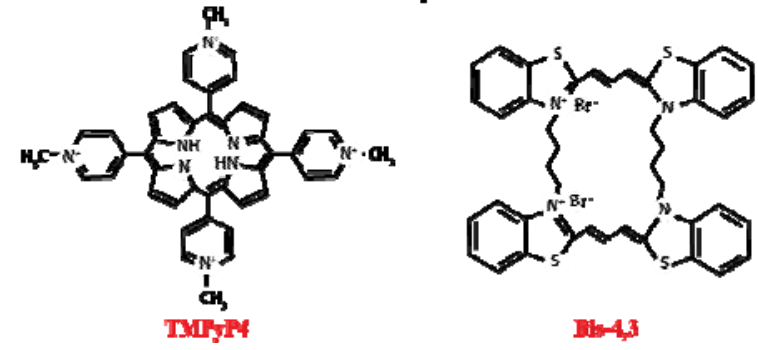

Figure 1. A, The localization of various G-rich regions of MTOR gene on chromosome 1 . B, the selected oligos from $5 \mathrm{~Kb}$ G-rich region. $\mathbf{C}$, the selected oligos from $8 \mathrm{~Kb}$ G-rich region. D, the selected oligos from 14Kb G-rich region; E, structure of TMPyP4, and $\mathbf{F}$, structure of Bis- 4,3 .
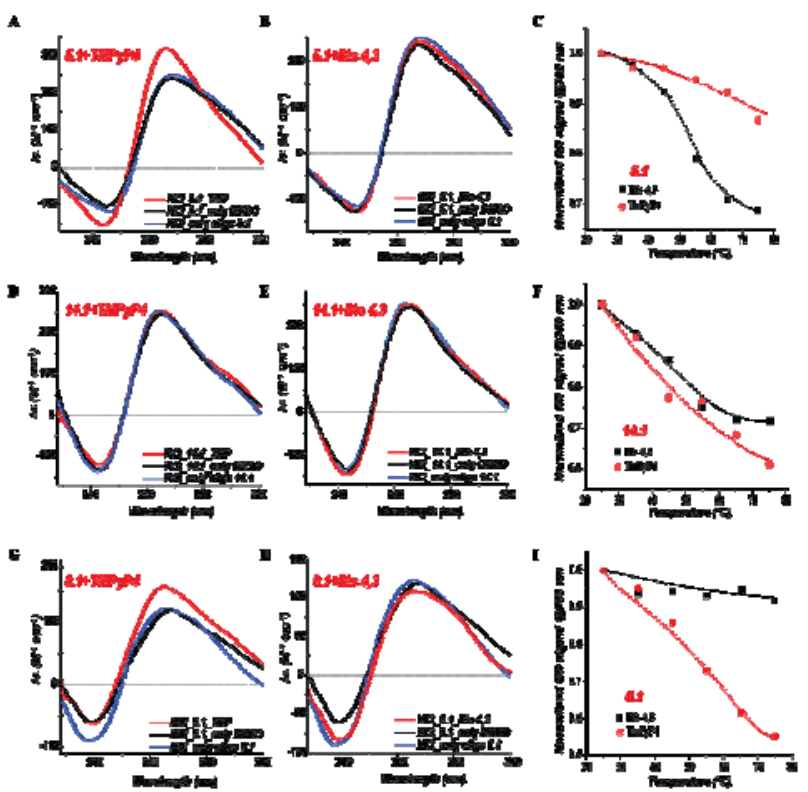

Figure 2. A, Comparative $\mathrm{CD}$ spectra of 5.1 oligo in presence $\mathrm{KCl}$ (black), $\mathrm{KCl}$ and DMSO (blue), and $\mathrm{KCl}$ and TMPyP4 (red). B, CD spectra of 5.1 oligo in presence $\mathrm{KCl}$ (black), $\mathrm{KCl}$ and DMSO (blue), and $\mathrm{KCl}$ and Bis-4,3 (red). C, CD melting curve of 5.1 oligo in presence of Bis-4,3 and TMPyP4, where it is normalized with the data point of $25^{\circ} \mathrm{C}$ at $260 \mathrm{~nm}$. D, CD spectra of 14.1 oligo in presence $\mathrm{KCl}$ (black), $\mathrm{KCl}$ and DMSO (blue), and $\mathrm{KCl}$ and TMPyP4 (red). E, $\mathrm{CD}$ spectra of 14.1 oligo in presence $\mathrm{KCl}$ (black), $\mathrm{KCl}$ and $\mathrm{DMSO}$ (blue), and $\mathrm{KCl}$ and Bis-4,3 (red). F, the $\mathrm{CD}$ melting curve of 14.1 oligo in presence of Bis-4,3 and TMPyP4, where it is normalized with the data point of $25^{\circ} \mathrm{C}$ at $260 \mathrm{~nm}$. G, CD spectra of 8.1 oligo in presence $\mathrm{KCl}$ (black), $\mathrm{KCl}$ and $\mathrm{DMSO}$ (blue), and $\mathrm{KCl}$ and TMPyP4 (red). $\mathrm{H}, \mathrm{CD}$ spectra of 8.1 oligo in presence $\mathrm{KCl}$ (black), $\mathrm{KCl}$ and DMSO (blue), and $\mathrm{KCl}$ and Bis-4,3 (red). I, the $\mathrm{CD}$ melting curve of 8.1 oligo in presence of Bis-4,3 and TMPyP4, where it is normalized with the data point of $25^{\circ} \mathrm{C}$ at $260 \mathrm{~nm}$. 
of G4 structures as well as providing insights on the topology or conformation of the secondary structures ${ }^{26-28}$. Six oligonucleotides were used according to the three G-rich regions [Fig. 1B, C, D]. In particular, the $5 \mathrm{~Kb}$ region yields three G-rich oligos (5.1, 5.2 and 5.3), 8Kb region possesses one G-rich oligo (8.1), and $14 \mathrm{~Kb}$ region contains two G-rich oligos (14.1 and 14.2). CD spectroscopy helped in assessing the secondary structures of the six sequences in presence of $\mathrm{KCl}$ [Fig. $\mathbf{S 2 A}$ ]. $\mathrm{CD}$ spectra of all oligos except 5.3 reveal signature bands of parallel quadruplexes, namely positive band at $260 \mathrm{~nm}$ and negative peak at $240 \mathrm{~nm}$ [Fig. S2]. Oligo 5.3 displays positive bands at $295 \mathrm{~nm}$ and $260 \mathrm{~nm}$ that are consistent with a predominantly mixed topology ${ }^{29}$. The effect of $\mathrm{LiCl}$ on formation of G4 by the six oligos was also assessed in comparison with the behaviour observed in $\mathrm{KCl}$ [Fig. S2B]. Interestingly, the presence of $\mathrm{LiCl}$ did not alter the $\mathrm{G} 4$ structure pattern for all the sequences. This reflects the inherent propensity and strength of G4 formation by the six oligos.

\section{G4 stabilization by TMPyP4 and a di-Carbocyanine ligand Bis-4,3}

The porphyrin-based ligand, TMPyP4 [Fig. 1E], has been widely deployed as a G4 stabilizing ligand ${ }^{30}$. We have previously reported the G4-binding behaviour of a novel dimeric carbocyanine dye, Bis-4,3 [Fig. 1F] that displayed strong propensity to selectively bind parallel G4 with concomitant fluorescence turn-on ${ }^{31,32}$. While our previous work focused on the G4-selective fluorescent reporting potential of Bis-4,3, interest in its ligand-like behaviour led to the formulation of the current work. Cytotoxicity assessment was essential before an investigation could be launched on the gene-regulatory potential of the small molecules. The cytotoxicity of both TMPyP4 and Bis-4,3 were measured by ROS activity in HeLa cells [Fig. S1]. Both TMPyP4 and Bis-4,3 were administered at $10 \mu \mathrm{M}$ concentration for 48 hours in HeLa Cells. Insignificant change in cytotoxicity for both the ligands was observed in comparison to control, thereby indicating that the duration of the dose $(10 \mu \mathrm{M}$ for $48 \mathrm{~h})$ is not harmful to in vivo cell model.

Among six oligos that had been identified as putative G4s in the MTOR gene, one each (5.1, 8.1 and 14.1) was chosen from the 5 $\mathrm{Kb}, 8 \mathrm{~Kb}$ and $14 \mathrm{~Kb}$ regions based on their propensity to form the parallel G4 structure [Fig. 1 E]. Both oligos 14.1 and 14.2 displayed CD bands that are consistent with the parallel G4 structure. CD has been widely used for probing the structural perturbation on G4s due to G4-selective ligands ${ }^{26-29}$. CD spectra of oligos 5.1 [Fig. 2 A, B], 8.1 [Fig. 2 D,E] and 14.1 [Fig. 2 $\mathbf{G , H ]}$ were measured in the separate presence of TMPyP4 and Bis-4,3. CD spectra of the oligos in presence of ligands clearly reveals the lack of structural distortions. In fact, the observed CD spectra for 5.1 in presence of TMPyP4 points to likely stabilization of the secondary structures. CD thermal melting experiments were used to further assess the effect of ligands on Grich oligos of MTOR gene. The CD melting curve of 5.1 oligo indicated greater stabilization in presence of TMPyP4 compared to Bis-4,3 [Fig. 2C]. In contrast, 8.1 showed more stabilization in Bis4,3 compared to TMPyP4 [Fig. 2I]. Both Bis-4,3 and TMPyP4 demonstrated a comparable stability for 14.1 oligo [Fig. 2F]. The $\mathrm{CD}$ melting experiments have revealed that the binding of TMPyP4 and Bis-4,3 to G4 sites are contingent on the oligo sequence and corresponding secondary structure.

\section{Primer extension assay to verify the presence of G-quadruplex.}

Primer extension assays have been used to demonstrate the presence of G-quadruplex secondary structures in a sequence of interest ${ }^{33}$. Based on the CD spectroscopic behaviour of oligo 5.1 in presence of the G4 binding ligands, we conducted the primer extension assay on oligo 5.1 as proof of concept of G4 formation [Fig. 3A]. This experiment was performed separately with the two ligands Bis-4,3 [Fig. 3B, C] and TMPyP4 [Fig. 3D,E]. Increasing concentrations of the G4-binding ligands was accompanied by decrease in full-length product formation as reflected from the gel electrophoretic pattern.

Interestingly, oligo 5.2 and 8.1 [Fig. S2] did not show a decrease in full-length product intensity. Nevertheless, the formation of truncated products in these instances indicates that the relevant quadruplex structures were stabilised to at the concentrations of ligands used for cellular studies. On the other hand, increasing concentrations of TMPyP4 on oligo 5.2 likely caused a shift in equilibrium from intermolecular quadruplex to an intramolecular quadruplex. The ability of TMPyP4 to cause a shift in equilibrium in vitro could portend a variability of ligand effect under cellular conditions.

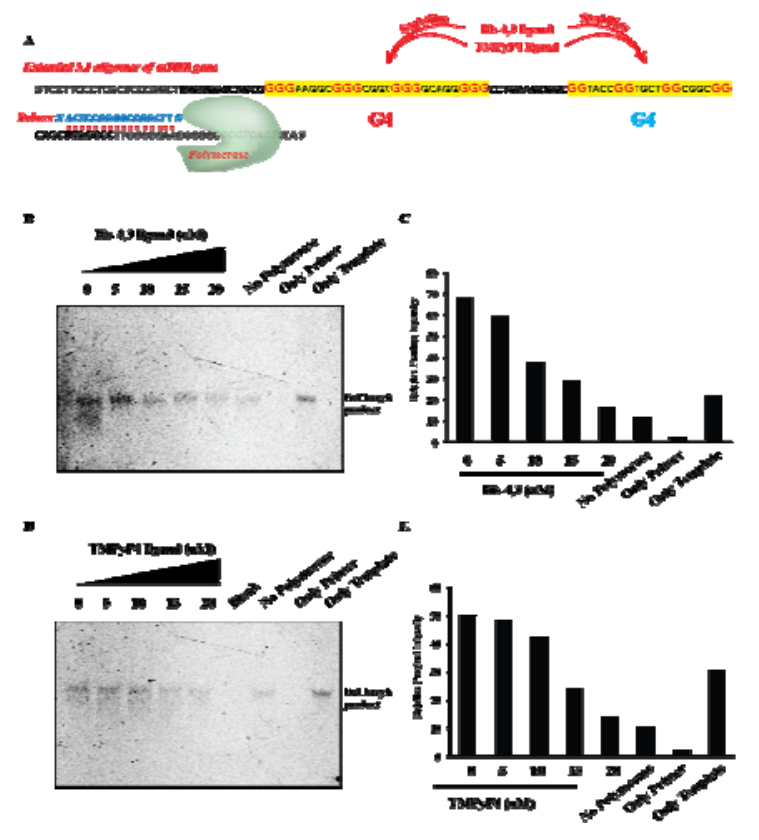

Figure 3. A schematic representation for primer extension assay for oligo 5.1. B, produced full length oligo after in vitro primer extension assay of 5.1 in presence of increasing amounts $(0,5,10,15$, and $20 \mathrm{uM})$ of Bis-4,3. C, graphical presentation of amount of full length product in presence of varying concentration of Bis-4,3. D, full length oligo generated after primer extension of 5.1 in presence of increasing amount $(0,5,10,15$, and $20 \mathrm{uM})$ of TMPyP4 in vitro. $\mathbf{E}$, graphical presentation of amount of full length product in presence of varying concentration of TMPyP4. 
These results verify the formation of G4s by G-rich regions in the upstream region of the MTOR gene. Further, they also highlight the possibility of using G4-stabilization as a method of regulating mTOR function. As discussed in subsequent sections, the ligand concentrations used caused an observable induction of autophagy in cellular studies. Thus, quadruplex structures formed in the mTOR gene could be the primary site for the ligand induced induction of autophagy.

\section{Influence of G4-targeting on mTOR activity in HeLa and SHSY-5Y cells}

The functional relevance of G4 vis-à-vis mTOR can be assessed by testing G4-selective ligands followed by western blots for the cognate proteins. We used two different cell lines, HeLa and SHSY-5Y, for this line of experimentation. The rationale behind using the two cancerous cell lines is that the cancerous condition generally shows higher basal levels of autophagy, which could

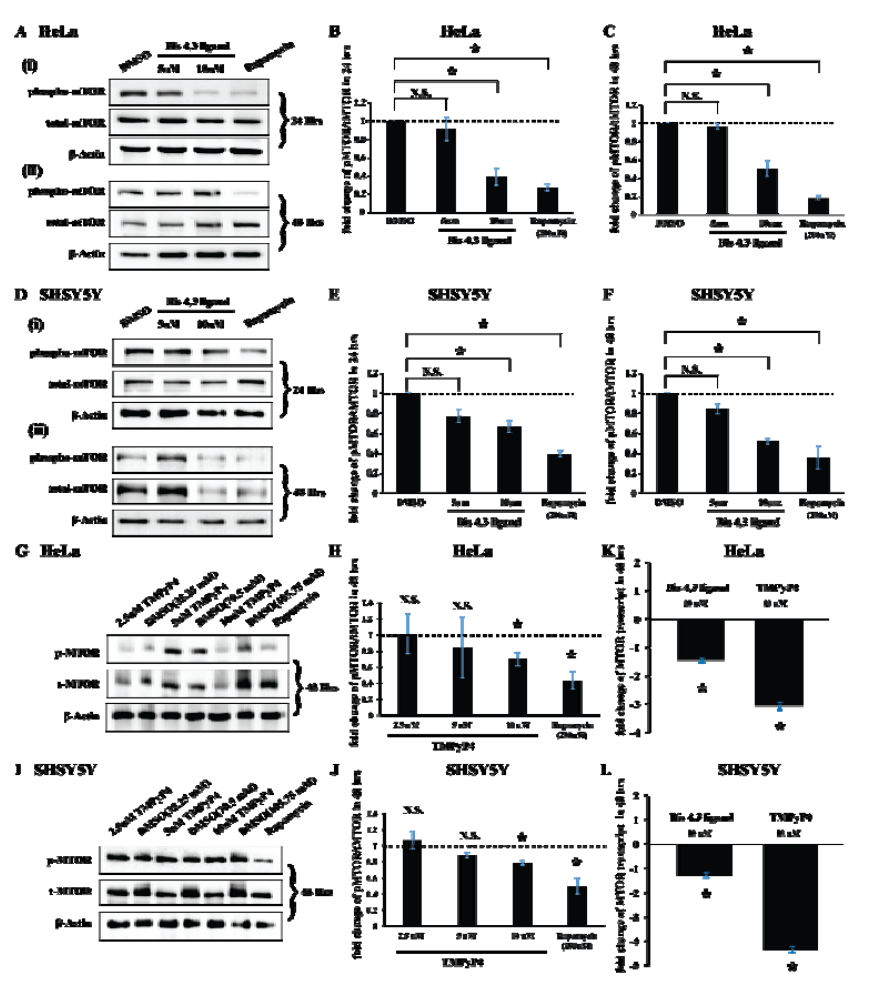

Figure 4. A, Western Blot of phospho-mTOR and total mTOR levels in HeLa cells after Bis-4,3 (5 and $10 \mu \mathrm{M})$ treatment for (i) 24 hours and (ii) 48 hours. Rapamycin treatment was used as positive control. B, graphical analysis of the phospho/total mTOR levels of HeLa cells after Bis-4,3 treatment of 24 hours. C, graphical analysis of the phospho/total mTOR levels of HeLa cells after Bis-4,3 treatment of 48 hours. D, Western Blot of phospho-mTOR and total mTOR level in SHSY-5Y cells after Bis-4,3 treatment for (i) 24 hours and (ii) 48 hours. Rapamycin treatment was used as positive control. E, graphical analysis of the phospho/total mTOR levels of SHSY-5Y cells after Bis-4,3 treatment for 24 hours. F, graphical analysis of the phospho/total mTOR levels of SHSY-5Y cells after Bis-4,3 treatment for 48 hours. G, Western Blot of phospho-mTOR and total mTOR level in HeLa cells after TMPyP4 treatment for 48 hours. Rapamycin treatment was used as positive control. H, graphical analysis of the phospho/total mTOR levels of HeLa cells after TMPyP4 treatment for 48 hours. I, Western Blot of phospho-mTOR and total mTOR level in SHSY-5Y cells after TMPyP4 treatment for 48 hours. Rapamycin treatment was used as positive control. J, graphical analysis of the phospho/total mTOR levels of SHSY-5Y cells after TMPyP4 treatment for 48 hours. $\mathbf{K}$, graphical representation of qRT-PCR $\Delta \Delta \mathrm{CT}$ values of mTOR fold change for both Bis-4,3 and TMPyP4 treated condition in HeLa cells for 48 hours compared to respective DMSO treated condition. L, graphical representation of qRT-PCR $\triangle \Delta \mathrm{CT}$ values of mTOR fold change for both Bis-4,3 and TMPyP4 treated condition in SHSY-5Y cells for 48 hours compared to respective DMSO treated condition. be easily monitored in presence of G4 stabilizing ligands. We first optimized the ligand effect of Bis-4,3 towards the expression of $\mathrm{mTOR}$ protein as indicated by western blots in HeLa and SHSY $-5 Y$ cells. 5 and $10 \mu \mathrm{M}$ of Bis-4,3 treatment for 24 hours was accompanied by no significant change in mTOR activity. mTOR activity was measured via the ratio of phosphor mTOR/total mTOR levels in HeLa and SHSY$5 \mathrm{Y}$ cells [Fig 4A(i), B, D(i), E]. Notably, $10 \mu \mathrm{M}$ treatment of Bis-4,3 for 48 hours significantly downregulated mTOR activity levels in both cell lines by 1.5 and 1.9 folds, respectively [Fig 4 A(ii), C, D(ii), F]. Considering the use of DMSO as solvent for the ligands, equal volume of DMSO was used as a control for the experiments. Further, the mTOR inhibitor rapamycin $(200 \mathrm{nM})$, was used as a positive control. Treatment with rapamycin for 48 hours significantly downregulated the mTOR activity by 2.8 and 3 fold in HeLa and SHSY-5Y cells, respectively.

Following the preliminary assessment of G4-selective ligand effect on mTOR activity, optimization of ligand concentrations was pursued by using different concentrations of ligands in both the cell lines. The optimum concentration of TMPyP4 was evaluated by using different concentrations of the ligand $(2.5 \mu \mathrm{M}, 5 \mu \mathrm{M}$, and $10 \mu \mathrm{M})$ for 48 hours in HeLa [Fig. 4G, H] and SHSY-5Y cells [Fig. 4I, J]. Based on western blot analysis, $10 \mu \mathrm{M}$ of TMPyP4 downregulated the mTOR activity in HeLa and SHSY-5Y cells by 1.4 folds and 1.27 folds, respectively in comparison to the DMSO control.

Next, mTOR transcript levels were measured in the presence of Bis-4,3 $(10 \mu \mathrm{M})$ and TMPyP4 $(10 \mu \mathrm{M})$ for 48 hours in HeLa [Fig. 4K] and SHSY-5Y [Fig. 4L]. The qRT-PCR data indicated significant downregulation of mTOR transcript level by 3 and 4-fold for HeLa and SHSY-5Y cells, respectively. The downregulated mTOR activity in both the cell lines corroborated our hypothesis that the G4stabilization of MTOR gene is capable of impeding expression of the mTOR protein.

\section{G-Quadruplex effect on autophagy activity in HeLa cells and SHSY -5Y cells.}

The mTOR protein is a potent regulator of several metabolic pathways in the cell ${ }^{15}$. One such catabolic pathway is autophagy, where the cell tries to sustain or maintain homeostasis by auto-digesting aberrant or unwanted proteins, lipids and cytosolic organelles under stress or starved condition. We studied the effect of G4 stabilizing ligands, Bis-4,3 and TMPyP4, on autophagy induction, in an attempt to implicate the direct involvement of G4 motifs of 
mTOR. It is widely known that the LC3B is the marker for autophagy induction ${ }^{34}$. An increased ratio between the lipidated (LC3BII) and cytosolic LC3B (LC3BI) signifies upregulation of autophagy, as compared to the basal level. First, the optimum ligand concentrations and incubation times for application of Bis-4,3 in HeLa cells were ascertained. As evident from the western blots, no significant change was observed in LC3BII levels compared to LC3BI levels upon 24 hour treatment with Bis-4,3 ligand

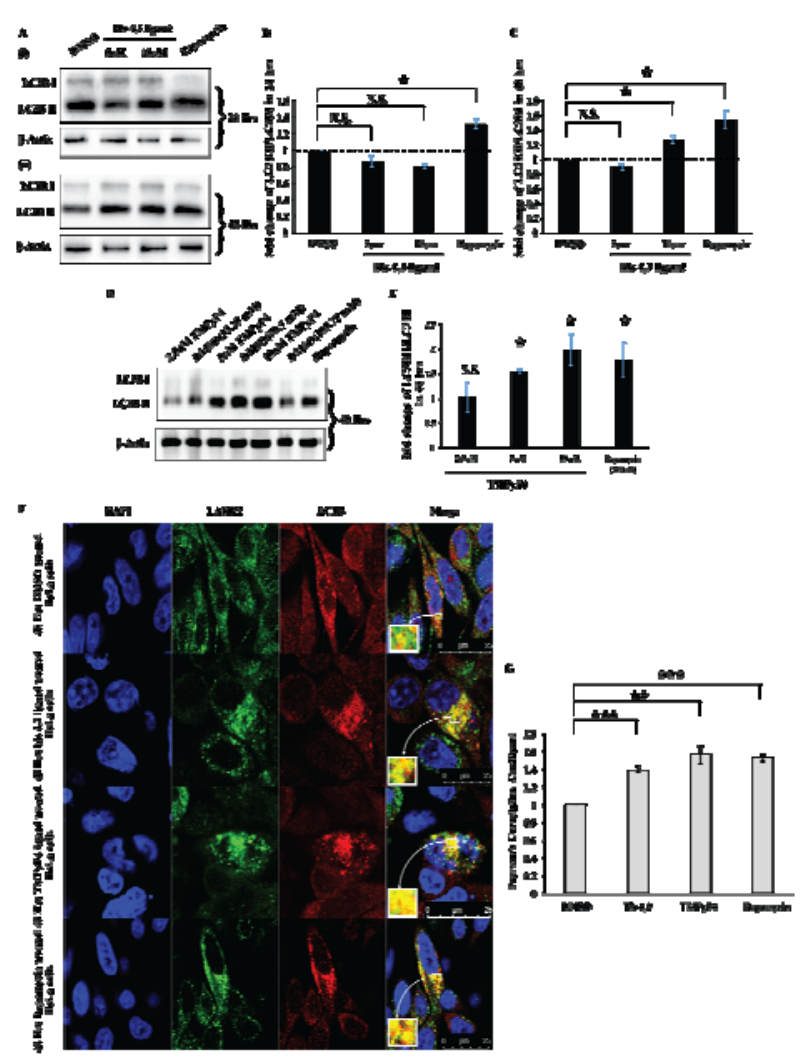

Figure 5: A, Western Blot of LC3B level in HeLa cells after Bis4,3 treatment for (i) 24 hours and (ii) 48 hours. Rapamycin treatment was observed as positive control. B, graphical analysis the LC3BII/LC3BI levels of HeLa cells after Bis-4,3 treatment for 24 hours. C, graphical analysis the LC3BII/LC3BI levels of HeLa cells after Bis-4,3 treatment for 48 hours. D, Western Blot of LC3B level in HeLa cells after TMPyP4 treatment for 48 hours. Rapamycin treatment was observed as positive control. E, graphical analysis of the LC3BII/LC3BI levels of HeLa cells after TMPyP4 treatment for 48 hours. F, Confocal images of DMSO, Bis-4,3 , TMPyP4 and rapamycin-treated HeLa cells. LAMP2 and LC3B proteins are indicated in green and red, respectively. Nucleus was stained with DAPI. G, graphical representation of Pearson's correlation co-efficient for the colocalization.
[Fig. 5A(i), B]. However, treatment with $10 \mu \mathrm{M}$ concentration of Bis-4,3 for 48 hours upregulated the LC3BII/LC3BI levels by 1.2 fold [Fig. 5A(ii), C]. Next, the optimum parameters for treatment with TMPyP4 were determined in analogous manner. We observed significant upregulation of LC3BII/LC3BI levels upon treatment with $5 \mu \mathrm{M}$ (1.6 fold) and $10 \mu \mathrm{M}$ ( 2 fold) concentrations of TMPyP4 for 48 hours [Fig. 5D, E]. Subsequently, we verified the autophagic flux by studying the fusion of autophagosomes with lysosomes via confocal microscopy. Verification of autophagy maturation or autophagic flux is essential, as the accumulation of autophagosomes is not always indicative of autophagy induction. The blockage of fusion between autophagosomes and lysosomes is considered as more substantive evidence of autophagy induction ${ }^{35}$. The confocal images of combined immunofluorescence for $\mathrm{LC} 3 \mathrm{~B}$ and the lysosome marker LAMP2A revealed that autophagosome efficiently colocalize with lysosomes in HeLa cells that had been treated with the G4 stabilizing ligands Bis-4,3 and TMPyP4 [Fig. 5F]. The extent of co-localization was measured by Pearson's correlation coefficient using Jacop plug-in in ImageJ software [Fig. 5G].

The LCBII/LC3BI levels were similarly determined for SHSY-5Y cells treated with both of the G4 targeting ligands. Treatment with Bis-4,3 for 24 hours was not accompanied by significant alterations in LC3B ratios [Fig. 6A(i), B]. However, treatment with $10 \mu \mathrm{M}$ of the ligand for 48 hours led to an elevated ratio by 1.47 fold [Fig. 6A(ii), C]. On the other hand, treatment of SHSY$5 \mathrm{Y}$ cells with $10 \mu \mathrm{M}$ of TMPyP4 for 48 hours substantially upregulated the LCBII/LC3BI levels by 2.3 fold as compared to the control DMSO treated condition [Fig. 6D, E]. There are no previously reported effects of TMPyP4 and Bis-4,3 on LC3BII/ LC3B1 ratios and mTOR expression levels. This work may thus be considered as the first study of G4 stabilization effect on mTOR levels. Confocal microscopy revealed autophagic flux for both Bis-4,3 and TMPyP4 treated SHSY-5Y cells based on the colocalization between LC3B and LAMP2A [Fig. 6F]. Colocalization coefficient was measured by Pearson's correlation coefficient [Fig. 6G].

The mTOR protein deficiency due to effect of G4-stabilizing ligands leads to upregulation of the LC3BII/LC3BI levels, which is an established marker for autophagy induction. Moreover, the co-localization between LC3B and LAMP2A implicated the autophagic flux for increase in LC3B levels. This observation precludes LC3B elevation due to blockage of autophagosome and lysosome fusion. Overall, the current work clearly highlights the molecular mechanism of G4 motifs in MTOR gene that can be leveraged towards induction of autophagy.

\section{Conclusions}


bioRxiv preprint doi: https://doi.org/10.1101/2021.09.26.461892; this version posted September 27, 2021. The copyright holder for this preprint (which was not certified by peer review) is the author/funder. All rights reserved. No reuse allowed without permission.

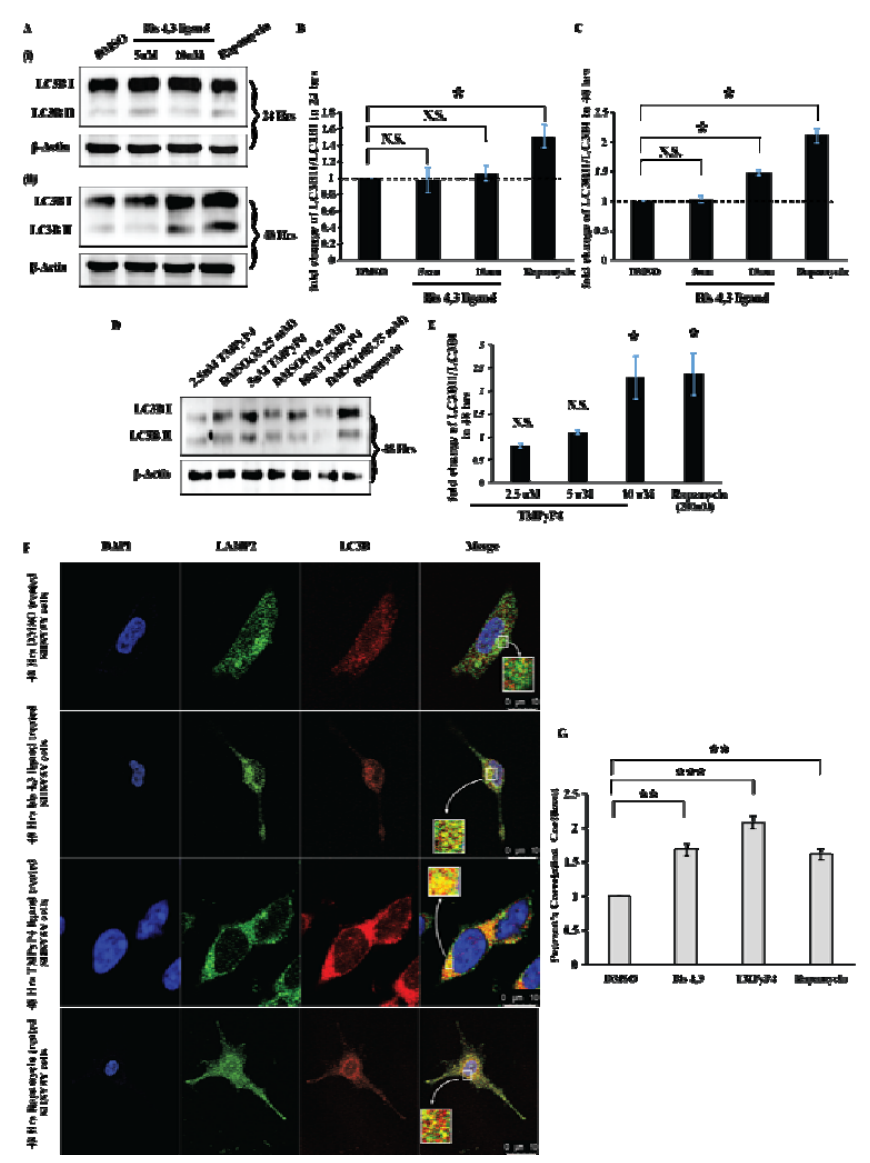

Figure 6: A, Western Blot of LC3B level in SHSY-5Y cells after Bis-4,3 treatment for (i) 24 hours and (ii) 48 hours. Rapamycin treatment was observed as positive control. B, graphical analysis the LC3BII/LC3BI levels of HeLa cells after Bis-4,3 treatment for 24 hours. C, graphical analysis the LC3BII/LC3BI levels of HeLa cells after Bis-4,3 treatment for 48 hours. D, Western Blot of LC3B level in HeLa cells after TMPyP4 treatment for 48 hours.

Rapamycin treatment was observed as positive control. E, graphical analysis of the LC3BII/LC3BI levels of HeLa cells after TMPyP4 treatment for 48 hours. F, Confocal images of DMSO, Bis-4,3, TMPyP4 and rapamycin-treated HeLa cells. LAMP2 and LC3B proteins are indicated in green and red, respectively. Nucleus was stained with DAPI. G, graphical representation of Pearson's correlation co-efficient for the colocalization.
This study has led to the following findings: (i) The upstream Grich regions of the MTOR gene have propensity to form Gquadruplex secondary structures. (ii) the G4 structures present in the upstream region of MTOR gene regulate its expression. (iii) the upstream G4 stabilization by small molecules TMPyP4 and Bis-4,3 stabilized the secondary structures, which in turn reduces the expression of mTOR protein. (iv) The reduced levels of mTOR perform a key role in catabolic metabolism through autophagy. Therefore, the G4-mediated regulation of mTOR presents a new therapeutic approach and can be investigated to control autophagy mediated cellular adaptation in diseases like cancer, type 2 diabetes and neurodegenerative diseases. It was recently demonstrated that PDS and BRACO-19 induced stabilization of G4-DNA structures in Atg7 gene leads to downregulation of autophagy in neurons ${ }^{36}$. While these may be contradictory to our reports, the observed induction of autophagy upon treatment with Bis-4,3 and TMPyP4 could likely be a consequence of the combination of the mTOR G4 structures, ligands and cell lines used in this study as well as other target sites that are yet to be identified.

Details pertaining to the relative importance of various G4-sites in the upstream region of mTOR are currently being worked on in our laboratory. 


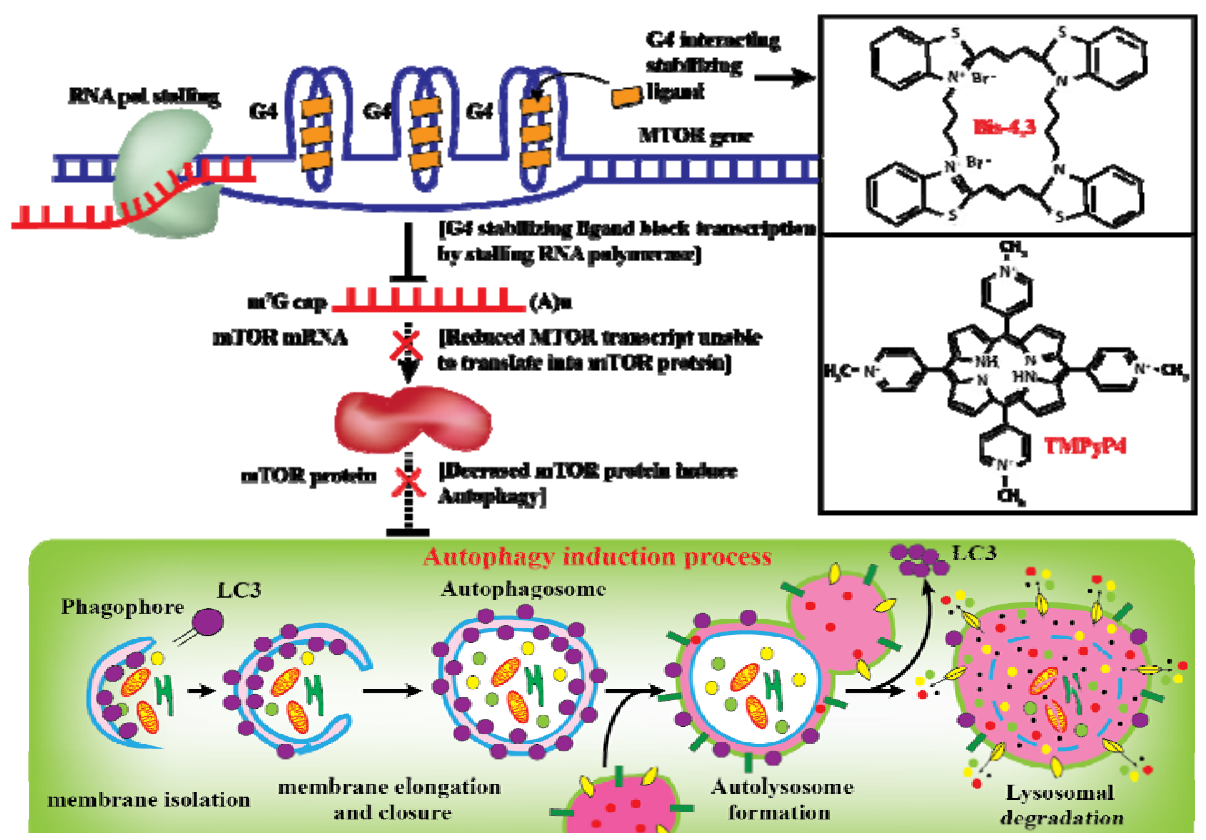

Figure 7: Schematic representation of G4 stabilization directed downregulation of mTOR triggers the autophagy

\section{Author Contributions}

Conceptualization: PM, BD; Data Curation; PM,CS, BD; Formal Analysis: PM, CS; Funding acquisition: PM, BD; Investigation: PM, CS; Methodology: PM, CS; Project administration: PM, BD; Resources: BD; Software: PM, CS; Validation: PM, CS; Writing-original: PM, CS, BD; Writing - review \& editing: BD.

\section{Conflicts of interest}

There are no conflicts to declare.

\section{Acknowledgements}

B.D. is grateful to IIT Gandhinagar for financial support of this work. P. M. would like to thank DST-SERB national post-doctoral fellowship (PDF/2018/000837). The authors would like to acknowledge inputs from Dr. Sharmistha Majumdar, Dr. Arnab Dutta and the Central Instrument Facility of IIT Gandhinagar

\section{Notes and references}


bioRxiv preprint doi: https://doi.org/10.1101/2021.09.26.461892; this version posted September 27, 2021. The copyright holder for this preprint (which was not certified by peer review) is the author/funder. All rights reserved. No reuse allowed without permission.

2 A. N. Lane, J. B. Chaires, R. D. Gray and J. O. Trent, Nucleic Acids Res., 2008, 36, 5482-5515.

3 G. Biffi, D. Tannahill, J. McCafferty and S. Balasubramanian, Nat. Chem., 2013, 5, 182-186.

4 E. Y. N. Lam, D. Beraldi, D. Tannahill and S. Balasubramanian, Nat. Commun., 2013, 4, 1796.

5 V. Pirota, M. Nadai, F. Doria and S. N. Richter, Molecules, , DOI:10.3390/molecules24030426.

6 S. Kumari, A. Bugaut, J. L. Huppert and S. Balasubramanian, Nat. Chem. Biol., 2007, 3, $218-221$.

7 S. Cogoi and L. E. Xodo, Nucleic Acids Res., 2006, 34, 2536-2549.

8 S. Rankin, A. P. Reszka, J. Huppert, M. Zloh, G. N. Parkinson, A. K. Todd, S. Ladame, S. Balasubramanian and S. Neidle, J. Am. Chem. Soc., 2005, 127, 10584-10589.

9 S. Asamitsu, S. Obata, Z. Yu, T. Bando and H. Sugiyama, Molecules, , DOI:10.3390/molecules24030429.

10 C. Marchetti, K. G. Zyner, S. A. Ohnmacht, M. Robson, S. M. Haider, J. P. Morton, G. Marsico, T. Vo, S. LaughlinToth, A. A. Ahmed, G. Di Vita, I. Pazitna, M. Gunaratnam, R. J. Besser, A. C. G. Andrade, S. Diocou, J. A. Pike, D. Tannahill, R. B. Pedley, T. R. J. Evans, W. D. Wilson, S. Balasubramanian and S. Neidle, J. Med. Chem., 2018, 61, $2500-2517$.

11 S. J. Welsh, A. G. Dale, C. M. Lombardo, H. Valentine, M. de la Fuente, A. Schatzlein and S. Neidle, Sci. Rep., 2013, 3, 2799.

12 J. Beauvarlet, P. Bensadoun, E. Darbo, G. Labrunie, B. Rousseau, E. Richard, I. Draskovic, A. Londono-Vallejo, J.-W. Dupuy, R. Nath Das, A. Guédin, G. Robert, F. Orange, S. Croce, V. Valesco, P. Soubeyran, K. M. Ryan, J.-L. Mergny and M. Djavaheri-Mergny, Nucleic Acids Res., 2019, 47, 2739-2756.

13 D. S. Arroyo, E. A. Gaviglio, J. M. Peralta Ramos, C. Bussi, M. C. Rodriguez-Galan and P. Iribarren, Int. Immunopharmacol., 2014, 18, 55-65.

14 P.-M. Wong, C. Puente, I. G. Ganley and X. Jiang, Autophagy, 2013, 9, 124-137.

15 R. A. Saxton and D. M. Sabatini, Cell, 2017, 168, 960-976.

16 S. K. Hindupur, A. González and M. N. Hall, Cold Spring Harb. Perspect. Biol., , DOI:10.1101/cshperspect.a019141.

17 G. Y. Liu and D. M. Sabatini, Nat. Rev. Mol. Cell Biol., 2020, 21, 183-203.

18 C. W. Yun and S. H. Lee, Int. J. Mol. Sci., , DOI:10.3390/ijms19113466.

19 D. Gozuacik and A. Kimchi, Oncogene, 2004, 23, 2891-2906.

20 M. Su, Y. Mei and S. Sinha, Role of the Crosstalk between Autophagy and Apoptosis in Cancer, https://www.hindawi.com/journals/jo/2013/102735/, (accessed November 3, 2020).

21 J. Karch, T. G. Schips, B. D. Maliken, M. J. Brody, M. A. Sargent, O. Kanisicak and J. D. Molkentin, eLife, , DOI:10.7554/eLife.30543.

22 W.-J. Zhou, R. Deng, X.-Y. Zhang, G.-K. Feng, L.-Q. Gu and X.-F. Zhu, Mol. Cancer Ther., $2009,8,3203-3213$.

23 N. I. Orlotti, G. Cimino-Reale, E. Borghini, M. Pennati, C. Sissi, F. Perrone, M. Palumbo, M. G. Daidone, M. Folini and N. Zaffaroni, Autophagy, 2012, 8, 1185-1196.

24 C. H. Jung, S.-H. Ro, J. Cao, N. M. Otto and D.-H. Kim, FEBS Lett., 2010, 584, 1287-1295.

25 O. Kikin, L. D’Antonio and P. S. Bagga, Nucleic Acids Res., 2006, 34, W676-W682.

26 J. Kypr, I. Kejnovská, D. Renciuk and M. Vorlícková, Nucleic Acids Res., 2009, 37, 1713-1725.

27 M. Vorlíčková, I. Kejnovská, K. Bednářová, D. Renčiuk and J. Kypr, Chirality, 2012, 24, 691-698.

28 M. Vorlíčková, I. Kejnovská, J. Sagi, D. Renčiuk, K. Bednářová, J. Motlová and J. Kypr, Methods San Diego Calif, 2012, 57, 64-75. 
29 H. Gattuso, A. Spinello, A. Terenzi, X. Assfeld, G. Barone and A. Monari, J. Phys. Chem. B, 2016, 120, 3113-3121.

30 E. Ruggiero and S. N. Richter, Nucleic Acids Res., 2018, 46, 3270-3283.

31 P. Chilka, P. R. Patlolla and B. Datta, RSC Adv., 2016, 6, 87400-87404.

32 P. Chilka, N. Desai and B. Datta, Molecules, , DOI:10.3390/molecules24040752.

33 R. Kumari, M. Nambiar, S. Shanbagh and S. C. Raghavan, PLoS ONE, , DOI:10.1371/journal.pone.0119722.

34 G. Runwal, E. Stamatakou, F. H. Siddiqi, C. Puri, Y. Zhu and D. C. Rubinsztein, Sci. Rep., $2019,9,10147$.

35 S. Nakamura and T. Yoshimori, J. Cell Sci., 2017, 130, 1209-1216.

36 J. F. Moruno-Manchon, P. Lejault, Y. Wang, B. McCauley, P. Honarpisheh, D. A. Morales Scheihing, S. Singh, W. Dang, N. Kim, A. Urayama, L. Zhu, D. Monchaud, L. D. McCullough and A. S. Tsvetkov, eLife, 2020,9 , e52283. 